04

\title{
Видеорегистрация долгоживущих плазмоидов вблизи объектов, подвергшихся дистанционному и непосредственному воздействию пинчевых сильноточных разрядов
}

\author{
(C) Б.Ю. Богданович, Н.В. Волков, Н.А. Лень, А.В. Нестерович
}

Национальный исследовательский ядерный университет „МИФИ“, 115409 Москва, Россия

e-mail: bybogdanovich@mephi.ru

(Поступило в Редакцию 29 июня 2018 г. В окончательной редакции 23 октября 2018 г.)

Приведены результаты наблюдения за плазмоидами, образующимися при периодическом разряде в потоке жидкости, в частности, воды, в окружающем пространстве в течение разряда и в послеразрядный период. Отмечено наличие долгоживущих светящихся образований не только в воздухе, но и на поверхности и над поверхностью близлежащих объектов. Проанализированы причины появления таких объектов и их свойств, имеющих признаки не только макро-, но и микрообъектов, таких как монополь Дирака.

DOI: 10.21883/JTF.2019.04.47304.257-18

\section{Введение}

Периодический разряд в потоке жидкости (ПРПЖ) известен уже более 20 лет [1], он нашел применение в ряде технологий, связанных с модификацией свойств металлических поверхностей [2-4]. Принципиальная схема установки показана на рис. 1. Основным устройством для подачи разряда является полый металлический наконечник 1. Обрабатываемый материал 3 располагается на столике 5 , который может перемещаться как автоматически, так и вручную. Наконечник 1 соединен с резервуаром жидкости 7 диэлектрическим трубопроводом 8 . На наконечник 1 подается постоянный отрицательный потенциал от блока питания 9 с постоянным напряжением порядка $7 \mathrm{kV}$. Образец 3 со столиком 5 находятся в диэлектрической камере 6 . Избыток жидкости сливается через систему стока 4. При зазоре 3-15 mm между образцом и наконечником возникает периодический разряд 2 с частотой повторения $f \approx 50-300 \mathrm{~Hz}$. Дуговой разряд длительностью несколько десятков наносекунд „точечно“ (диаметр кратера 5-20 m) воздействует на обрабатываемую поверхность. Скорость перемещения столика изменялась в пределах от 0.25 до $2.0 \mathrm{~mm} / \mathrm{s}$.

Процесс разряда приводит к распаду сплошности жидкой массы и возникновению ударных волн, сопровождающихся мощным треском и выбросами части вещества на отдаленное (в масштабе эксперимента) расстояние (до десяти сантиметров и более).

Свойства ПРПЖ как инструмента физических исследований изучены еще не достаточно полно. Ключом к пониманию физических процессов, сопровождающих разряд, может явиться анализ процессов плазмообразования как на стадии протекания разряда, так и в послеразрядный период. Фото- и киносъемка разряда показала, что он имеет две характерные стадии - разряд по поверхности струи жидкости и пинчевые разряды, существующие как совместно с основным (рис. 2), так и самостоятельно. Шаровидные плазмоиды наблюдаются как в объеме, так и на поверхности электрода в отсутствие основного разряда (рис. 3, 4). Обнаружены светящиеся нити длиной до сантиметра, концы которых имеют утолщение. Продолжительность их существования, как правило, не превышает единиц секунд.

Видео- и фотосъемка разряда с расстояния $1 \mathrm{~m}$ и более показала наличие светящихся плазмоидов, покидающих область разряда со скоростью более $25 \mathrm{~m} / \mathrm{s}$ (рис. 5).

На рисунке отмечены три четких изображения плазмоида, соответствующие, по нашему мнению, трем значениям температуры плазмы, а именно белое (макси-

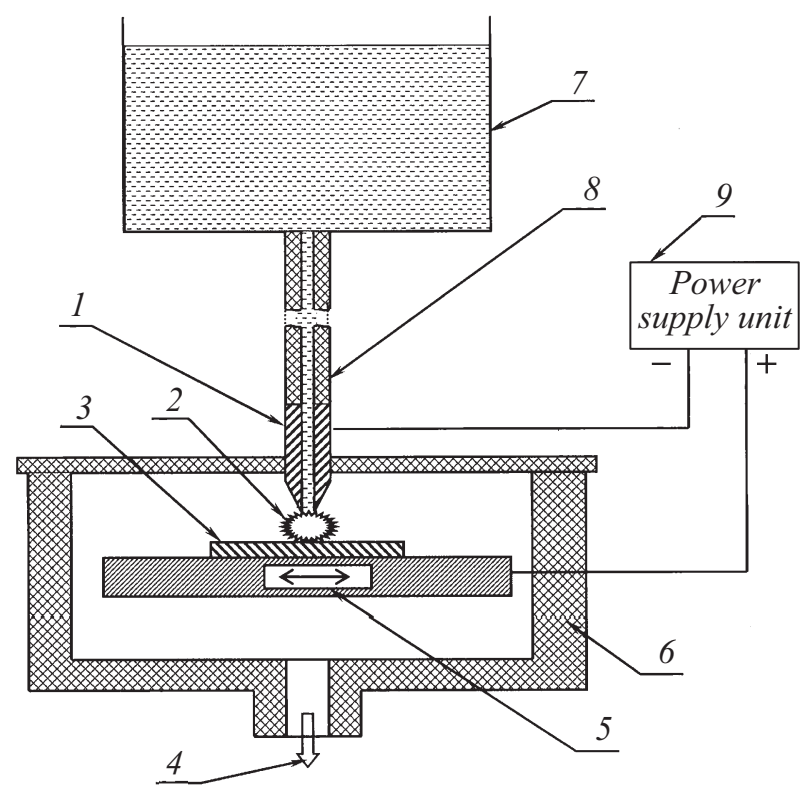

Рис. 1. Схема установки для обработки образцов ПРПЖ: 1 - наконечник; 2 - область разряда; 3 - образец; 4 система стока жидкости; 5 - столик; 6 - диэлектрическая камера; 7 - резервуар с жидкостью; 8 - диэлектрический трубопровод; 9 - блок питания. 


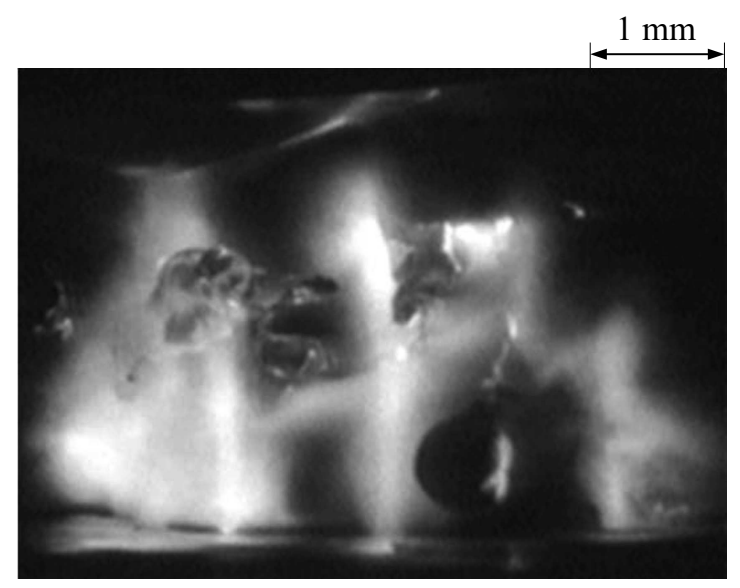

Рис. 2. Характерный вид пинчевых разрядов.

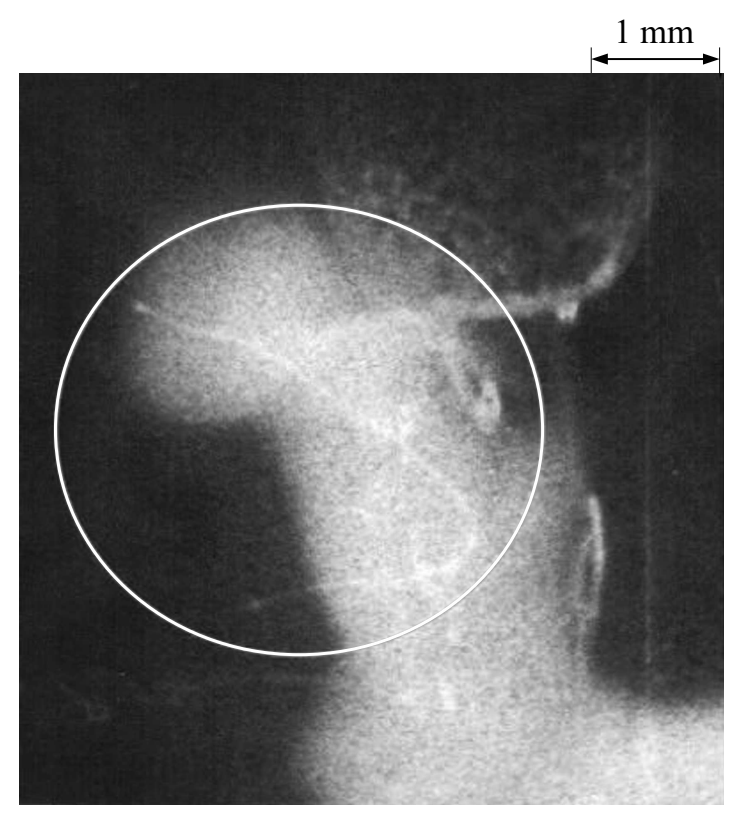

Рис. 3. Шаровидный плазмоид и светящаяся нить в разряде (обведена кругом).

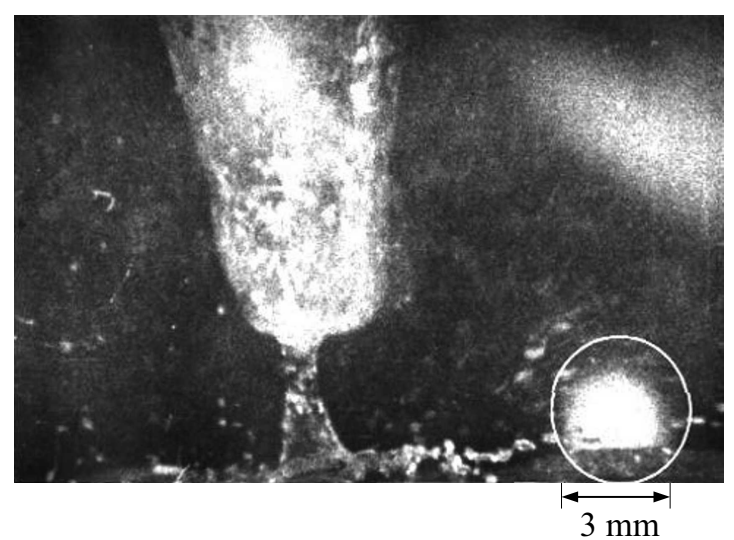

Рис. 4. Шаровидный плазмоид на поверхности заземленного электрода в послеразрядный период (обведен кругом).

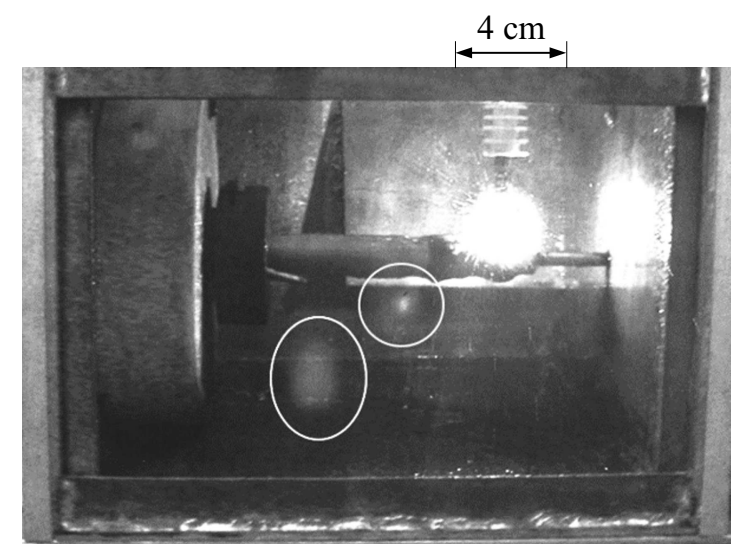

Рис. 5. Светящийся плазмоид в разные моменты движения от разряда. Белым кругом выделено изображение движущегося плазмоида в разных стадиях.

мальная температура) - при образовании плазмы в разряде, голубое и красное по мере остывания в воздухе и увеличения размеров при движении. Красный цвет соответствует длине волны в диапазоне 6200-7600 А, голубой - 4800-5100 А̊. Ускорение плазмы может быть обусловлено как ударными волнами при сильноточном разряде, сопровождающимися громким звуком, так и электрическим полем на высоковольтном электроде (до $7.5 \mathrm{kV}$ ).

\section{Регистрация долгоживущих плазмообразований}

Изучение поверхности металлических электродов, подвергшихся воздействию ПРПЖ, обнаружило наличие светящихся точечных зон на поверхности, свечение которых имеет достаточно большую длительность (более двух суток) [1].

Для выяснения природы наблюдаемых явлений и динамики процессов появления светящихся зон было проведено постоянное наблюдение за поверхностями как подвергнутыми воздействию, так и находящимися в зоне, непосредственно прилегающей к разряду, а также в непосредственном контакте с такими поверхностями, что могло бы подтвердить предположения о существовании стабильных частиц, ответственных за длительное свечение (плазмообразование) на микро- и макроуровнях.

Анализ полученных данных показал наличие светящихся объектов, совершающих движение по поверхности. Форма объектов - предположительно тороидальная, ось тороида параллельна поверхности. Имеются основания считать их вращающимися относительно этой оси. Тороиды неоднородны по азимуту, о чем свидетельствует движение затемненного сектора слева направо и наоборот, что, вероятнее всего, соответствует вращению тороида вокруг собственной оси (рис. 6). Они имеют 


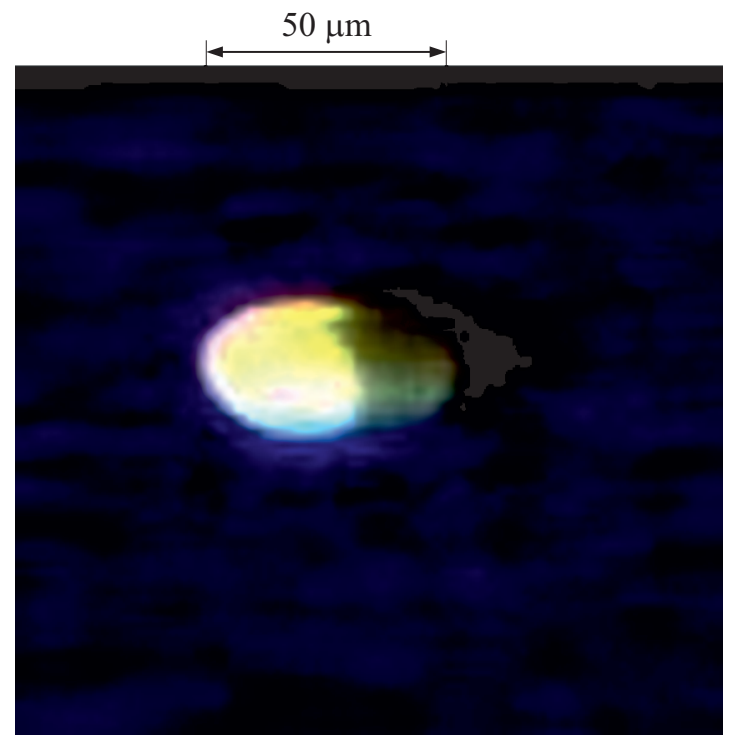

Рис. 6. Светящийся тороидальный объект, совершающий поступательное и вращательное движение над поверхностью электрода.

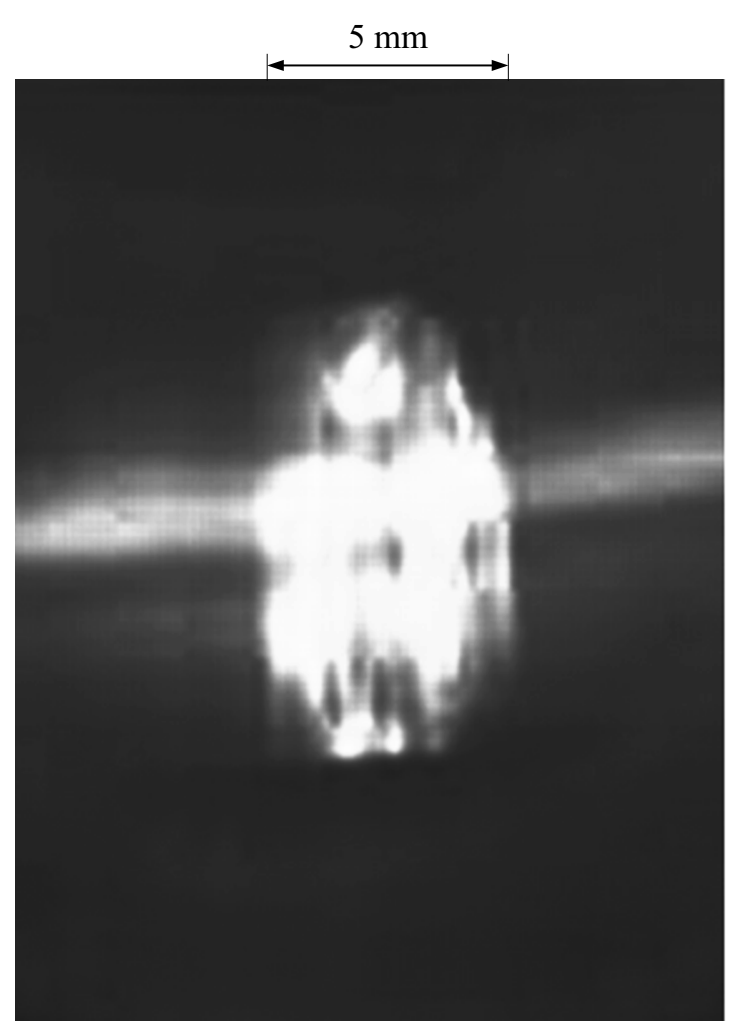

Рис. 7. Поток электронов из диэлектрической поверхности и формируемый им сложный объект типа светящихся колец, вращающихся вокруг собственной общей оси, совершающий „качение“ по поверхности.

размеры $\sim 50 \mu \mathrm{m}$, скорость - более $10 \mu \mathrm{m} / \mathrm{s}$. Время их нахождения в поле зрения микроскопа с видеокамерой - несколько десятков секунд. Цвет излучения плазмоида - зеленый (что может свидетельствовать о температуре воздуха, соответствующей длине волны 5100-5500 А). На отдельных объектах данного вида темных секторов не просматривается.

По предварительным оценкам, скорость вращения тороида - порядка 0.5 оборота в секунду.

В процессе наблюдения за состоянием поверхности из прозрачного диэлектрика, находящегося над поверхностью металлической пластины, расположенной ранее в непосредственной близости от ПРПЖ во время формирования пинчей, было установлено следующее.

Из поверхности эмитируется поток частиц (предположительно электронов), вызывающих свечение воздуха (аналогичная картина наблюдается после эмиссии электронов из источника или инжектора электронов через фольгу). Через 10-20 s этот поток формируется в набор нескольких колец (5 или 6) одинакового диаметра, вращающихся вокруг одновременно собственной и общей оси, параллельной плоскости (горизонтальной) (рис. 7).

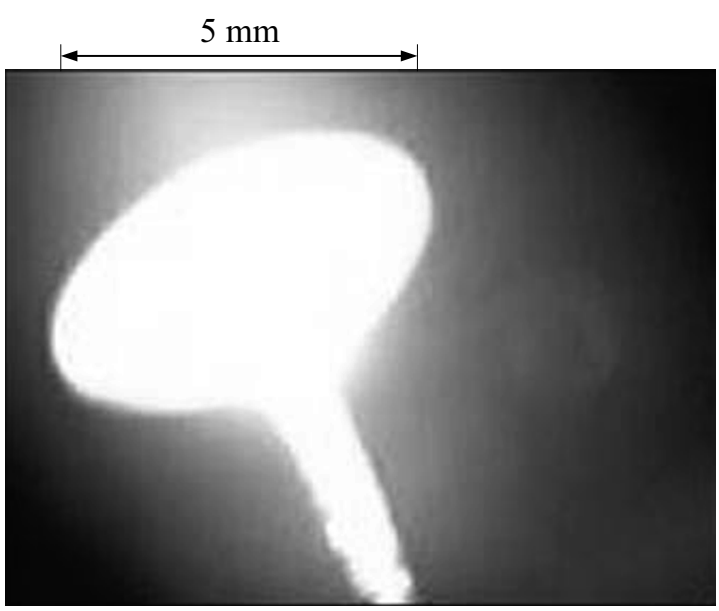

Рис. 8. Взаимодействие плазмоида с диэлектрической поверхностью.

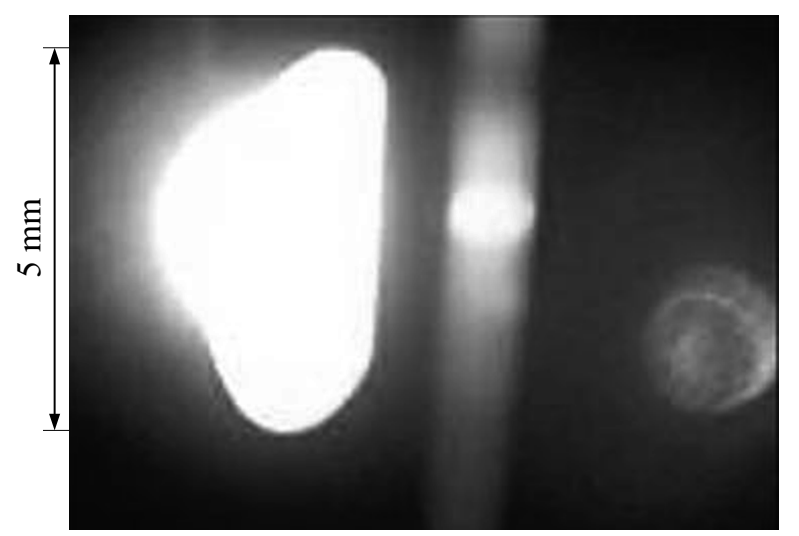

Рис. 9. Отрыв части плазмоида от основной части. Просматривается объект более слабого свечения с другой стороны пластины (справа внизу). 
Вся система начинает вращаться вокруг общей оси и перемещается в горизонтальной плоскости. Затем она трансформируется в некий электронный смерч, вращающийся вокруг вертикальной оси и по своей форме и размерам практически тождественный пинчам в разряде. Высота - несколько миллиметров (не более 10) Этот „смерч“ удаляется от видеокамеры и исчезает. Весь процесс от начала и до конца длится около $1 \mathrm{~min}$. Поперечный размер вращающегося „смерча“ не более $5 \mathrm{~mm}$.

Не менее важной и информативной является видеорегистрация взаимодействия пинчевых плазмоидов с диэлектрической поверхностью (рис. 8).

Светящийся объект в форме эллипсоида, вращающегося вокруг своей оси, зависает над поверхностью, и в своей нижней части, ближайшей к поверхности, образует вытянутый в сторону поверхности „протуберанец“, который затем отрывается и проходит сквозь поверхность. Особый интерес вызывает наличие с другой стороны поверхности, в которую втягивается отпочковавшаяся часть эллипсоида, шаровидного объекта, совершающего перемещение в пространстве, на первый взгляд, мало скоррелированное с движением эллипсоида, однако находящееся в пределах поля зрения видеокамеры. Размеры эллипсоида и шаровидного объекта - несколько (5-6) mm. Длительность процесса наблюдения - около 1 min. Цвет свечения - голубой (рис. 9).

\section{Обсуждение результатов}

Существование квазистационарных, хотя и относительно короткоживущих, светящихся плазмоидов сферической и линейной конфигурации обосновано теоретически авторами и коллегами в рамках общей теории относительности (ОТО) с привлечением понятий о микрогравитации и искривления пространств-времени [5]. Показано, что стационарное состояние, в принципе, возможно при соответствующей концентрации ионов вещества в объеме плазмоида, в частности, при параметрах пинчевых разрядов в проведенных экспериментах.

Дальнейший анализ указывает на целесообразность привлечения понятий о стационарных центрах формирования и стабилизации плазмоидов, в частности, стабильных частиц типа магнитного диполя [6], ответственных за вращение компонентов плазменных образований (точнее говоря, типа „дионов“ [7]). На это указывает наличие светящихся утолщений на концах „нити“ в области локализации разряда. На возможность включения в область рассматриваемых явлений указано различными исследователями, в частности в [8].

Приведенные в настоящей работе результаты указывают на необходимость более тщательного исследования данного фактора в природе общеизвестных явлений.

Ключевым остается вопрос о появлении магнитных монополей (или объектов с аналогичными свойствами) в зоне разряда. Одним из объяснений этого процесса может явиться регистрация на рентгеновской пленке отпечатков, указывающих на наличие в ближайшей к разряду зоне объектов, по форме и свойствам принадлежащих к данному типу физических объектов [9], в частности, их проникновение через двойные конверты защиты фотопленки и вид отпечатка.

В соответствии с современными представлениями [8] в поле монополя электроны могут образовывать куперовские пары и вращаться, стремясь к монополю, но теряя при этом энергию в ближней к нему зоне.

Вокруг монополя может образовываться „ореол“ из электронов и атомов ионизованного воздуха. В совокупности с полем „шлейфа“ общее магнитное поле может интерпретироваться как поле диполя, во внешней зоне которого могут стабильно существовать электроны, в том числе находящиеся в квантовом состоянии при сверхнизкой температуре, и образовывать структуры, тождественные тем, которые показаны на рис. 7. Вращение вокруг общей оси напоминает динамику электронов в магнитном поле Земли (градиентный дрейф и т. п.).

Анализ полученных результатов однозначно указывает на такие свойства наблюдаемых объектов и явлений, как наличие сверхтекучести, способность образовывать объекты, обладающие четко фиксированными размерами, вращение объектов как целое и в отдельных частях.

Именно магнитные монополи (или „дионы“), сопровождаемые шлейфом куперовских электронных пар, могут создавать магнитные поля дипольной конфигурации, в которых электроны внешней оболочки образуют электронные кольца одинакового размера в силу квантовости магнитного потока.

Результаты проникновения плазмоида сквозь диэлектрическую преграду могут объясняться тем, что „электронный шлейф“ следует за монополем (или, точнее, кластером, содержащим ионы, электроны и дионы), проникающим предположительно сквозь данную преграду.

Работа выполнена в рамках Соглашения №17-72-20228 между Российским научным фондом и Национальным исследовательским ядерным университетом „МИФИ“ о предоставлении гранта на проведение фундаментальных и поисковых научных исследований.

В обеспечении фото- и видеосъемки участвовали Пучков А.Н. и Новожилов Б.М.

\section{Список литературы}

[1] Алферов П.В., Богданович Б.Ю., Волков Н.В., Лень Н.А., Нестерович А.В., Старостин А.Н., Сильнов С.М. // Инженерная физика. 2000. № 4. С. 19-22.

[2] Bogdanovich B.Y., Kalin B.A., Nesterovich A.V. // Appl. Research. 2016. Vol. 7. N 5. P. 673-681.

[3] Гавзе А.Л., Шестаков И.И., Нестерович А.В., Богданович Б.Ю., Алферов П.В. // Металлобработка. 2003. Т. 3. № 15. C. 8-11. 
[4] Гавзе А.Л., Матевосьян А.П., Нестерович А.В., Богданович Б.Ю. // Металловедение и термическая обработка металлов. 2001. № 9. С. 34-39.

[5] Богданович Б.Ю., Нестерович А.В., Суханова Л.А., Хлестков Ю.А. Описание пинчевых плазмоидов в рамках общей теории относительности. Известия вузов. Физика. 2016. T. 59. № 10. C. 109-119.

[6] Родионов Б.У. Гипотетические магнитные монополи в атмосфере Земли. ДАН. 1996. Т. 350. № 5. С. 684-686.

[7] Сборника статей „Монополь Дирака“ / Под ред. Б.М. Болотовского, Ю.Д. Усачева. М.: Изд-во Мир, С. 40-58.

[8] Вишневский Р.П. Сверхпроводимость монополя Дирака. Электронный ресурс. Режим доступа:

http://lightdynamics.narod.ru/Sverhprovodimost_monopolya_Dirka.htm Дата обращения: 20.06.2018.

[9] Нестерович А.В., Богданович Б.Ю., Буянов Г.О. Экспериментальное исследование ионизации окружающей среды в зоне периодического разряда в потоке жидкости (ПРПЖ). IV Междунар. конф. Лазерные, плазменные исследования и технологии. Лаплаз-2018: Сборник научных трудов. М.: НИЯУ МИФИ, 2018. 543 с. 